\title{
The Relationship Between Bore Resonance Frequencies and Playing Frequencies in Trumpets
}

\author{
P. Eveno ${ }^{1) *}$, J.-F. Petiot ${ }^{2)}$, J. Gilbert ${ }^{3)}$, B. Kieffer ${ }^{1)}$, R. Caussé ${ }^{1)}$ \\ 1) Institut de Recherche et Coordination Acoustique/Musique (UMR CNRS 9912), 1 place Igor Stravinsky, \\ 75004 Paris, France. pauline.eveno@mail.mcgill.ca \\ 2) Institut de Recherche en Communications et Cybernétique de Nantes (UMR CNRS 6597), 1 rue de la Noë, \\ BP 92101, 44321 Nantes Cedex 3, France. \\ 3) Laboratoire d'Acoustique de l'Université du Maine (UMR CNRS 6613), Avenue Olivier Messiaen, \\ 72085 Le Mans Cedex 9, France.
}

\begin{abstract}
Summary
The aim of this work is to study experimentally the relationship between the resonance frequencies of the trumpet, extracted from its input impedance, and the playing frequencies of notes, as played by musicians. Three different trumpets have been used for the experiment, obtained by changing only the leadpipe of the same instrument. After a measurement of the input impedance of these trumpets, four musicians were asked to play the first five regimes of the instrument, for four different fingerings. This was done for three dynamic levels and repeated three times. Statistical methods were implemented to assess the variability in the playing frequencies, and to study quantitatively their relationships with the bore resonance frequencies. A limited influence of the musician on the instrument overall intonation is observed, as well as a weak influence of the dynamic levels on the pitch of the notes. The results show that for most of the regimes, variations of the resonance frequency lead to same order variations of the playing frequency of the corresponding note. We noticed also that the sum function, derived from the input impedance, does not give a better prediction of the playing frequency than the input impedance itself.
\end{abstract}

\section{Introduction}

Measuring and computing wind musical instruments input impedance is now well mastered $[1,2,3,4,5,6]$. As part of a larger project aimed toward helping instrument makers to design and characterise their musical instruments, this work focuses on how the bore resonance frequencies, taken from the input impedance, can be related to the playing frequencies. Indeed, instrument makers are primarily interested in the overall intonation of their instruments in playing situations, and therefore they need some predictive indicators.

Some studies attempt to find a solution to this issue by taking the coupling between the instrument and the musician into account. The case of reed instruments is treated

\footnotetext{
* New affiliation:

Computational Acoustic Modeling Laboratory, Schulich School of Music of McGill University, 555 Sherbrooke Street West, Montréal, Canada. pauline.eveno@mail.mcgill.ca
}

by Gilbert et al. [7] and Farner et al. [8] by using the harmonic balance technique adapted to self-sustained oscillations of wind instruments such as clarinets. The resonator (i.e. the instrument body) is the linear part, treated in frequency domain, while the driving system (the reed) is the nonlinear part, treated in time domain. The harmonic balance technique can also be used for brass instruments [9]. Three control parameters representing the "virtual" musician have to be defined: the pressure inside the mouth, the resonance frequency of the lips, and the inverse of lips mass density. Depending on the choice of these parameters, it is possible to obtain a series of playing frequencies, such as those obtained by the musician. The coupling between the musician and the instrument can also be investigated using a simplified model in which a single mechanical lip mode is coupled to a single mode of the acoustical resonator, as done by Cullen et al. [10] for the trombone. It is also possible to predict the intonation of the instrument by synthesizing the notes it can produce. Many studies are carried out on physical modelling using temporal methods $[11,12]$.

For the saxophone, for some advanced performance techniques (bugling and altissimo playing), musicians can 
use the resonance of their vocal tract to play a note close to a weak bore resonance, or even decrease the sounding pitch to several semitones below the standard pitch for the same fingering $[13,14,15]$. It seems that this technique is not used by trumpet players $[16,17]$.

The musician has a significant role in determining the playing frequencies, this aspect being difficult to take into account. Therefore, the first aim of this paper is to determine an order of magnitude of the brass player's influence on the overall intonation of the instrument. Then, it aims at finding some objective indicators from the input impedance which can predict the playing frequencies without taking the musician's behaviour into account, as intended by Pratt and Bowsher [18] and previously by Wogram [19]. This will be done by recording a large number of notes played by several musicians on three trumpets.

Section 2 presents some basic information about the acoustics of the trumpet. Section 3 describes the recording of notes played by the musicians on the different trumpets and the analysis of the data. From these measurements, an analysis of the musicians' behaviour is presented in section 4 . In section 5, the playing frequencies of the recorded notes are compared to the bore resonance frequencies taken from the input impedance of the trumpets. Section 6 presents the data first as a normal distribution and then, in order to minimize the influence of the musician on the results, it focuses on frequency differences instead of the frequencies themselves. Finally, the relevance of the sum function [19], a function made from the input impedance to predict the intonation, is discussed.

\section{Trumpet resonances and playing fre- quencies: Preliminary discussion}

Campbell and Greated [20] as well as Fletcher and Rossing [21] give a large overview on brass instruments. A summary about trumpets is reported here as well as a discussion on the coupling with the musician.

The acoustic response of an instrument at different frequencies can be characterised by its input impedance (impedance computed or measured at the input of the entire instrument, that is to say at the input plane of the mouthpiece). A typical input impedance of a brass instrument (see Figure 1) shows a large number of bore resonance frequencies, where the impedance amplitude is maximum and the phase is passing through zero. Some of these resonance frequencies are associated with a note (or oscillation regime) that the musician can play. In the example of Figure 1, corresponding to the basic fingering of a $\mathrm{B} b$ trumpet where all the three valves are up, the resonances 2 to 6 correspond to the series of concert notes $\mathrm{Bb} 3$, $\mathrm{F} 4, \mathrm{Bb} 4, \mathrm{D} 5, \mathrm{~F} 5$ (harmonic series of $\mathrm{Bb}$ ). The first resonance does not correspond to a normally playable note on the trumpet. The three valves offer height combinations, which allow the construction of the whole chromatic scale, since the activation of a valve produces an elongation of the air column which lower the resonance frequencies of the instrument. The first valve brings down the frequency

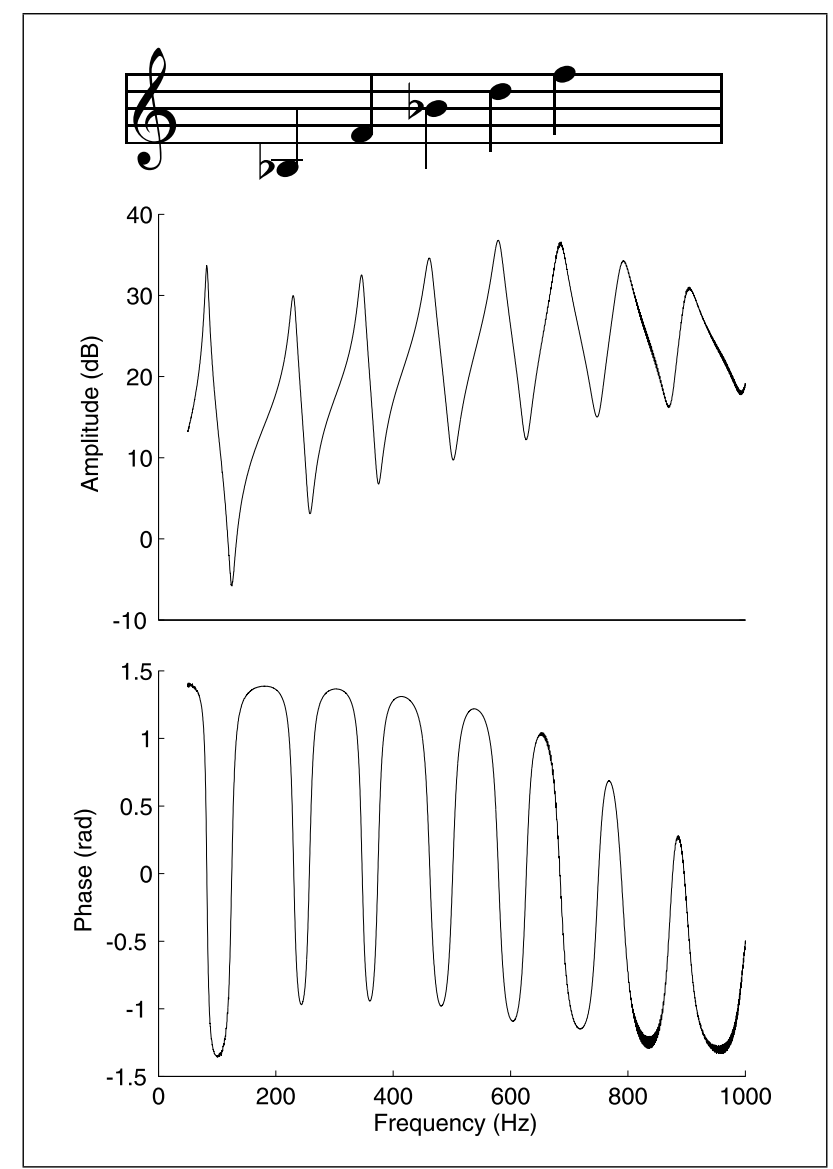

Figure 1. Measurement of the input impedance amplitude (in dB) and phase (in rad) of the trumpet called NORM with all the three valves up, with the notes corresponding to each impedance peak above (concert pitch of a $\mathrm{B} b$ trumpet). The trumpet and the set-up used for the measurement are presented in Section 3.1.

of one tone, the second of a semitone, and the last one of one and a half tones. In the rest of the article, a pressed valve will be noted 1 and a valve up will be noted 0 .

An initial estimation of the instrument intonation can be carried out by comparing the bore resonance frequencies to their corresponding notes in the equally-tempered scale, as it is shown in Figure 2 for the trumpets called CHMQ, DKNR and NORM in this study. These instruments are presented in section 3. This representation is often used by instruments makers and is for example presented in the BIAS software ${ }^{1}$. These graphs show that the series of resonances of the trumpet can be considered as harmonic with a $[-20,+20]$ cents precision interval (the resonance frequencies are almost aligned on an horizontal line), apart from the resonances corresponding to the second regime of 100, 110 and 111 fingerings that are too low. These diagrams also show that, by increasing the length of the bore, the resonance frequencies become more distant from the frequencies of their corresponding notes in the

\footnotetext{
${ }^{1}$ BIAS is the Brass Instrument Analysis System developed at the Institute of Music Acoustics in Vienna: http://www.bias.at/?page_id= $5 \&$ sprache $=2$.
} 

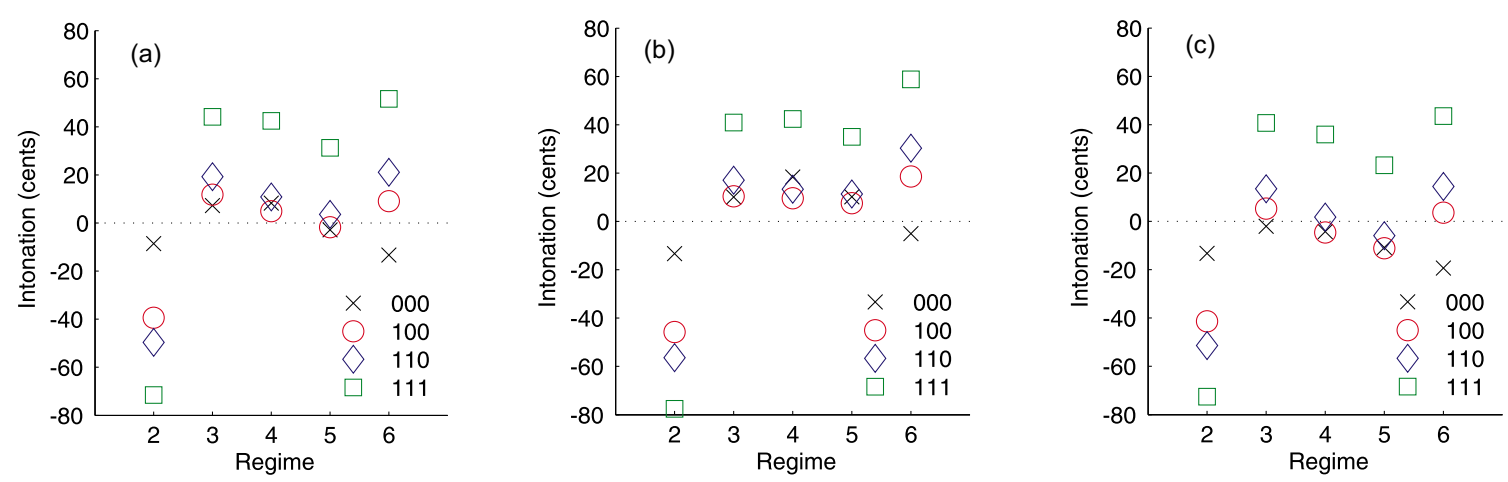

Figure 2. Intonation graph of trumpet (a) CHMQ, (b) DKNR and (c) NORM, obtained by calculating the difference in cents between each resonance frequency of the input impedance of each trumpet for the four fingerings and its corresponding note in the equallytempered scale.

equally-tempered scale. Consequently, if we assume that the resonance frequencies are representative of the playing frequencies, notes should be easier to play in tune with the 000 fingering than with the 111 fingering.

However, this affirmation has to be considered cautiously because this graph forgets an important element of the trumpet playing: the musician. Indeed, these diagrams are only estimations of the intonation because the playing frequencies are not exactly equal to the bore resonance frequencies. The differences between those frequencies result from a complex aeroelastic coupling between the lips of the musician and the resonator. Thus, the intonation of the instrument is not only controlled by the closest resonance frequency but possibly conditioned by upper resonance frequencies of the resonator [22].

Furthermore, a wind instrument is not an instrument with a fixed sound, that is to say the musician can modify the pitch and the timbre of the played note by controlling his/her embouchure and "bending" the notes. The embouchure represents the capacity of the musician to control the mechanical parameters of his/her vibrating lips, by modifying his/her facial musculature as well as the support force of the lips on the mouthpiece. This also includes the ability to control the air flow between the lips.

\section{Set-Up and Data Analysis}

\subsection{Set-Up}

A parametrised leadpipe, made of four different interchangeable parts, was designed [23, 24] as it is shown in Figure 3. Several parts with various values for the radii $r_{1}$, $r_{2}, r_{3}$ and $r_{4}$ were manufactured with a numerically controlled turning machine. A letter has been given to each part of the leadpipe, corresponding to the dimensions of the radii. Thus, using the same $\mathrm{B} b$ trumpet (Bach model Vernon, bell 43) with the same mouthpiece (Bach 1 1/2 C) and the parametrised leadpipe, different instruments with small different acoustical behaviours can be designed. Three leadpipes were considered for the study: the two

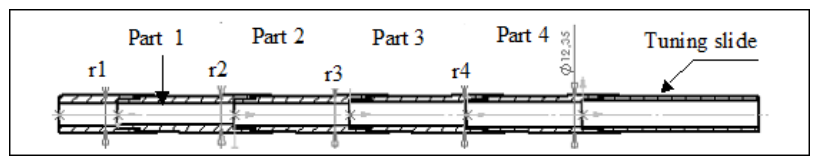

Figure 3. Parametrisation of the leadpipes used in this work (from Petiot et al. [24]). Radii $r_{1}, r_{2}, r_{3}$ and $r_{4}$ are given in Table I.

leadpipes presented in the Table I and a leadpipe originally provided with the trumpet, called NORM, as in "normal leadpipe". These three instruments are all playable and are, at first sight, very similar from a musical point of view. For all the tests, the position of the tuning slide was similar: it was pulled out of a length of $1 \mathrm{~cm}$ for all the trumpets and all the musicians.

The input impedances for these trumpets were then measured for four different fingerings $(000,100,110$ and 111) using a set-up described by Macaluso and Dalmont [4]. The first regime is not played with a trumpet. In this study, the notes will thus be recorded for regimes 2 to 6 with these four fingerings. Nevertheless, some of these notes do not correspond to the usual fingerings used by the musician. Regimes 2 to 6 are normally played by musicians for fingerings 000 and 100 . For the 110 fingering, musicians play notes from the second regime to the fifth. The sixth is generally not used since concert pitch D5 can be played with the fifth regime of the 000 fingering. For the 111 fingering, only regimes 2 and 3 are usually played. Regimes 4, 5 and 6 are an alternative way of playing the notes $\mathrm{E} 4, \mathrm{G} \sharp 4$ and $\mathrm{B} 4$, for which musicians usually use the third regime of the 010 fingering (E4), the fourth regime of the 100 fingering $(\mathrm{G} \sharp 4)$ and the fifth regime of the 110 fingering (B4). These fingerings have been chosen in order to study the whole range of the trumpet frequencies, from the lowest pitch to the highest. Furthermore, while certain combinations of regimes and fingerings are almost never used by musicians, it has been interesting to include them in this study. Indeed, trumpet players are not used to playing these notes so there is no "learning effect", which 
Table I. Description of the two parametrised leadpipes used in this study (the radii are given in $\mathrm{mm}$ ).

\begin{tabular}{|c|ccccccccc|}
\hline & \multicolumn{3}{|c}{ Part 1 } & \multicolumn{2}{c}{ Part 2 } & \multicolumn{2}{c}{ Part 3 } & \multicolumn{2}{c|}{ Part 4 } \\
\hline & $r_{1}$ & $r_{2}$ & $r_{2}$ & $r_{3}$ & $r_{3}$ & $r_{4}$ & $r_{4}$ & $r_{5}$ \\
CHMQ & 4.64 & 5 & 5 & 5.5 & 5.5 & 5.7 & 5.7 & 5.825 \\
DKNR & 4.64 & 5.45 & 5.45 & 5.5 & 5.5 & 5.825 & 5.825 & 5.825 \\
\hline
\end{tabular}

means that they are more likely to play without focusing on the intonation.

Four musicians, one professor at a music school and three experienced amateurs, were asked to play the three trumpets to record the sounds. After a short warm-up, each trumpet player had to play the five first playable notes (regimes 2 to 6) by saying the name of the note before playing, in order to have a short rest between the notes and "forget" the pitch of the previous note. Indeed, trumpet players are interested in testing the flexibility of their instrument and, if necessary, they bend the note in order to correct the intonation defects. Nevertheless, the task for the musician is different here since it consists of letting the instrument guide him, even if it means playing out of tune. The musicians were then asked to play the note with the easiest emission, without trying to correct the intonation. These recordings were made for three dynamic levels in order to study their influence on the playing frequencies: first mezzo forte, then piano, and finally forte. Afterwards, each trumpet player had to move to the next fingering with the same protocol and so on for the four fingerings and the three trumpets. They had to repeat the whole process three times in order to test their reproducibility. Finally, 4 trumpet players times 3 trumpets times 4 fingerings times 5 regimes times 3 dynamic levels times 3 repetitions give 2160 notes to analyse.

\subsection{Data Analysis}

The playing frequency of the notes has been analysed with the YIN [25] software ${ }^{2}$, which is an estimator of the fundamental frequency specially calibrated for speech and music. Overlapping square windows of $68 \mathrm{~ms}$ length were used. This is more than twice the largest expected period for all the measured notes. We noticed that musicians were not able to play a perfectly steady note, and slight oscillations around the playing frequency were observed. Figure 4 shows an example of the frequency evolution of one note, concert $\mathrm{Bb} 3$, played by a musician with the basic fingering (000) at mezzo forte. At the beginning, the frequency rapidly increases: this is a typical transient. The same effect is happening during the quiescent. After removing the transient and the quiescent, a quasi-stationary part stays, where the frequency is fluctuating a few hertz. Therefore, from a measured signal like the one in Figure 4, what we call in this paper the playing frequency, will be determined as the mean of the instantaneous frequency during the time $t$ of the quasi-stationary part. The

\footnotetext{
${ }^{2}$ It can be downloaded at http: //audition.ens.fr/adc/
}

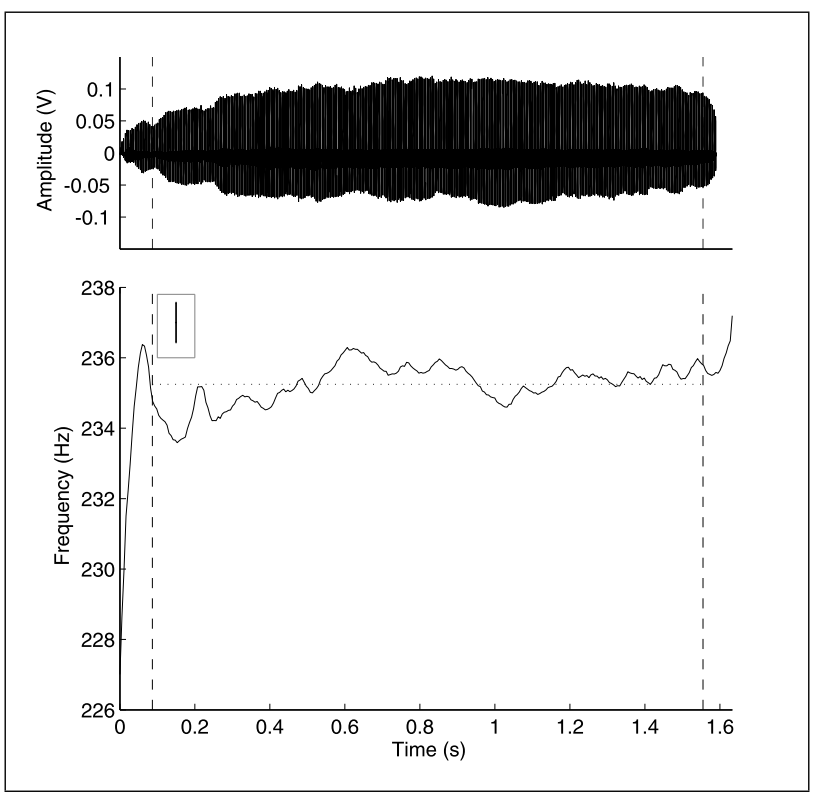

Figure 4. Waveform (above) and evolution of the frequency of the $\mathrm{B} b 3$ note played by a musician with the basic fingering (000) at mezzo forte. Dashed lines separate, on the left the transient and on the right the quiescent, from the rest of the quasi-stationary signal. The dotted line represents the mean value of the frequency during this quasi-stationary part. The error bar in the grey rectangle at the top left-hand corner represents twice the standard deviation of the frequency during the quasi-stationary signal.

standard deviation is then calculated in order to estimate the ability of the trumpet player to play at a stable playing frequency.

The measurements of the trumpets' input impedances and the recording of the musicians were carried out at different temperatures. The input impedance was measured at $23^{\circ} \mathrm{C}$ whereas notes were played at a room temperature of $25^{\circ} \mathrm{C}$. According to Gilbert et al. [26] and Noreland [27], we consider that the temperature of the air column was around $28^{\circ} \mathrm{C}$ during playing. Consequently, for a reliable comparison, resonance frequencies need to be moved forward from the equivalent temperature shift. Since resonance frequencies of both cones and cylinders are proportional to the sound velocity, which can be written as $c=331.45 \sqrt{T / T_{0}} \mathrm{~m} / \mathrm{s}$ with $T$ the temperature in Kelvin and $T_{0}=273.16 \mathrm{~K}$, it can be considered that the resonance frequencies of a trumpet are proportional to the square root of the temperature expressed in Kelvin. Consequently, the resonance frequencies from the measured input impedances are increased by 14 cents which is the equivalent of $5^{\circ} \mathrm{C}$ in order to be at the same level of the playing frequencies' temperature. 
Finally, the frequency of the resonances is precisely determined with a peak fitting technique using a least square method on the complex impedance [28]. This method represents the impedance in the Nyquist plot. In this plot, the resonance is locally a circle that should go through the experimental points. Then, the resonance frequency is the angle of the point, which is the furthest from the origin. This method is the one used by Macaluso and Dalmont [4] and leads to an estimation of the resonance frequency with an uncertainty of about 5 cents. The resonance frequency could also be determined with the phase zero crossing. Nevertheless, as explained in [29, p. 149], the amplitude of the impedance gives more information about the tuning and the ease of playing than the phase, that is why this definition of the resonance frequency was chosen.

\section{Musicians' behaviours}

\subsection{Descriptive analysis of the playing frequencies}

In order to study the behaviour of each musician, we represent by a boxplot the differences (in cents) between each playing frequency and its respective resonance frequency for all the notes played. A boxplot is a convenient way of graphically representing a distribution of numerical data through their five-number summaries: the smallest observation (sample minimum), lower quartile $\left(25^{\text {th }}\right.$ percentile, bottom of the box), median, upper quartile $\left(75^{\text {th }}\right.$ percentile, top of the box), and largest observation (sample maximum).

One boxplot per dynamic level allows one to study the influence of the dynamic level on the playing frequencies. In each boxplot of Figure 5, there are 180 notes which correspond to 3 trumpets times 4 fingerings times 5 regimes ( 2 to 6 ) times 3 attempts.

These boxplots show that the playing frequencies are, on average, higher than the bore resonance frequencies and that the four musicians play at a slight higher pitch at the piano dynamic level (p) than at mezzo forte ( $\mathrm{mf}$ ) or forte (f). The dynamic leads to less than a 10 cents difference on the playing frequency median. The four trumpet players have a similar behaviour as they all play, on average, in the order of 8 to 20 cents above bore resonance frequencies. These results could be consistent with a dominant outward striking regime of oscillations for the lips that has been observed in previous studies [30, 31, 32]. Nevertheless, Figure 5 shows that musicians can also play below the resonance frequencies for some notes. Players can thus have different behaviours depending on the note they play, on their embouchure, etc. and a single mechanical oscillator cannot model the complete behaviour of the lip reed [33, 34, 35, 10].

\subsection{Modelling of the playing frequency with ANOVA}

In order to estimate the influence of each controlled factor of the experiment on the playing frequency, it can be modelled using the analysis of variance method (ANOVA)

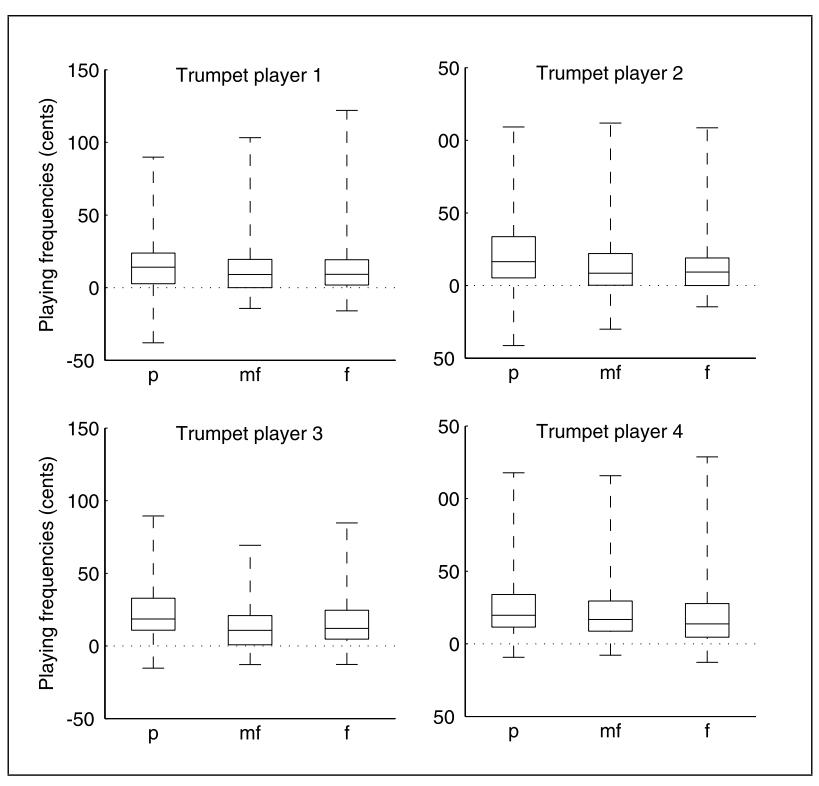

Figure 5. Boxplots representing the statistics of the playing frequencies of the notes played by each player for the three dynamic levels ( $\mathrm{p}, \mathrm{mf}$ and $\mathrm{f}$ ). Data are expressed in cents, as a difference between each playing frequency and its corresponding resonance frequency.

$[36,37]$. ANOVA is a collection of statistical models to model a quantitative variable (the response) with qualitative variables (the factors). It belongs to the general frame of the linear model, and proposes statistical tests to determine whether or not the means of different groups of data are all equal, in the case of more than 2 groups (generalisation of the t-test).

In our application, the response is the playing frequency, which is supposed to be modelled as the sum of different qualitative factors (independent variables). The general model in the case of a five-factors ANOVA is given by

Fplay $_{i j k l m}=\mu+\alpha_{i}+\beta_{j}+\gamma_{k}+\delta_{l}+\eta_{m}+\varepsilon_{i j k l m}$

with

- $\mu$ a constant,

- $\alpha_{i}$ the effect of the level $i$ of the musician ( $i=1$ to 4 since there are four musicians),

- $\beta_{j}$ the effect of the level $j$ of the dynamic level $(j=1$ to 3 since there are three dynamics),

- $\gamma_{k}$ the effect of the level $k$ of the trumpet $(k=1$ to 3 since there are three trumpets),

- $\delta_{l}$ the effect of the level $l$ of the fingering ( $l=1$ to 4 since there are four fingerings),

- $\eta_{m}$ the effect of the level $m$ of the regime ( $m=1$ to 5 since there are five regimes),

- and $\varepsilon_{i j k l m}$ the error term.

Each coefficient represents the influence of the level of the factor on the response. From the measurements, a least square procedure is used to estimate these coefficients (minimization of the squared error between the measured playing frequency and the playing frequency given by the 
Table II. Results of the ANOVA model for all the data of the study. Source means "the source of the variation in the data", DF means "the degrees of freedom in the source", SS means "the sum of squares due to the source", MS means "the mean sum of squares due to the source", F means "the F-statistic" and P means "the $p$-value".

\begin{tabular}{|cccccc|}
\hline Source & DF & SS & MS & F & P \\
\hline Musician & 3 & $1.7 \mathrm{e} 3$ & $5.8 \mathrm{e} 2$ & 1.6 & 0.184 \\
Dynamic & 2 & $2.0 \mathrm{e} 3$ & $1.0 \mathrm{e} 3$ & 2.8 & 0.063 \\
Trumpet & 2 & $1.8 \mathrm{e} 1$ & 8.9 & 0.02 & 0.976 \\
Fingering & 3 & $4.9 \mathrm{e} 6$ & $1.6 \mathrm{e} 6$ & $4.5 \mathrm{e} 3$ & $<0.0001$ \\
Regime & 4 & $4.6 \mathrm{e} 7$ & $1.1 \mathrm{e} 7$ & $3.1 \mathrm{e} 4$ & $<0.0001$ \\
\hline
\end{tabular}

model). A classical F-test is used to assess the significance of the effect of the factors. The sources can be considered to have a significant impact on the data if the probability $p$ is lower than 0.05 [36]. Table II gives the results of the ANOVA model, the last column indicating the probabilityvalue $p$ of the F-test (false rejection probability).

Only two factors have a significant effect on the playing frequency: the fingering and the regime $(p<0.0001)$. Changing the fingering or the regime leads to important modifications of the playing frequencies, which is obvious. The effects of the trumpet, the musician and the dynamic level are not significant at the 5\% level. It means that the influence of these factors on the playing frequency is very weak. An analysis of the coefficients shows that the piano dynamic level leads, on average, to a slightly higher playing frequency than the mezzo forte and forte dynamic levels. Moreover, it indicates that the first trumpet player plays, on average, slightly lower than the other three. However these effects are negligible compared to those of the fingering and the regime. Furthermore, an analysis of variance with interactions terms between each pair of factors shows that interactions are not significant.

\section{Playing frequencies vs Resonance fre- quencies}

\subsection{Visualisation of the raw data}

Figure 6 presents the playing frequencies of notes $\left(\mathrm{F}_{\text {play }}\right)$ as functions of the bore resonance frequencies $\left(\mathrm{F}_{\text {res }}\right)$ of the corresponding regimes. Both of these frequencies are expressed in cents taking the equally-tempered scale as a reference. In every subfigure,there are two error bars at the upper left-hand corner. The error bar on the left represents twice the average standard deviation of a note, called $\sigma_{1}$. Indeed, as explained in section 3.2, each played note is determined by an average frequency (which corresponds to the playing frequency taken into account in the paper) and a standard deviation $\sigma$. The error bar thus stands for the average $\sigma$ over all the played notes, which is equal to 5 cents. The error bar on the right represents twice the average reproducibility of the trumpet players, called $\sigma_{2}$. Indeed, each musician will repeat 9 times the same note ( 3 dynamic levels times 3 attempts). The standard deviation is

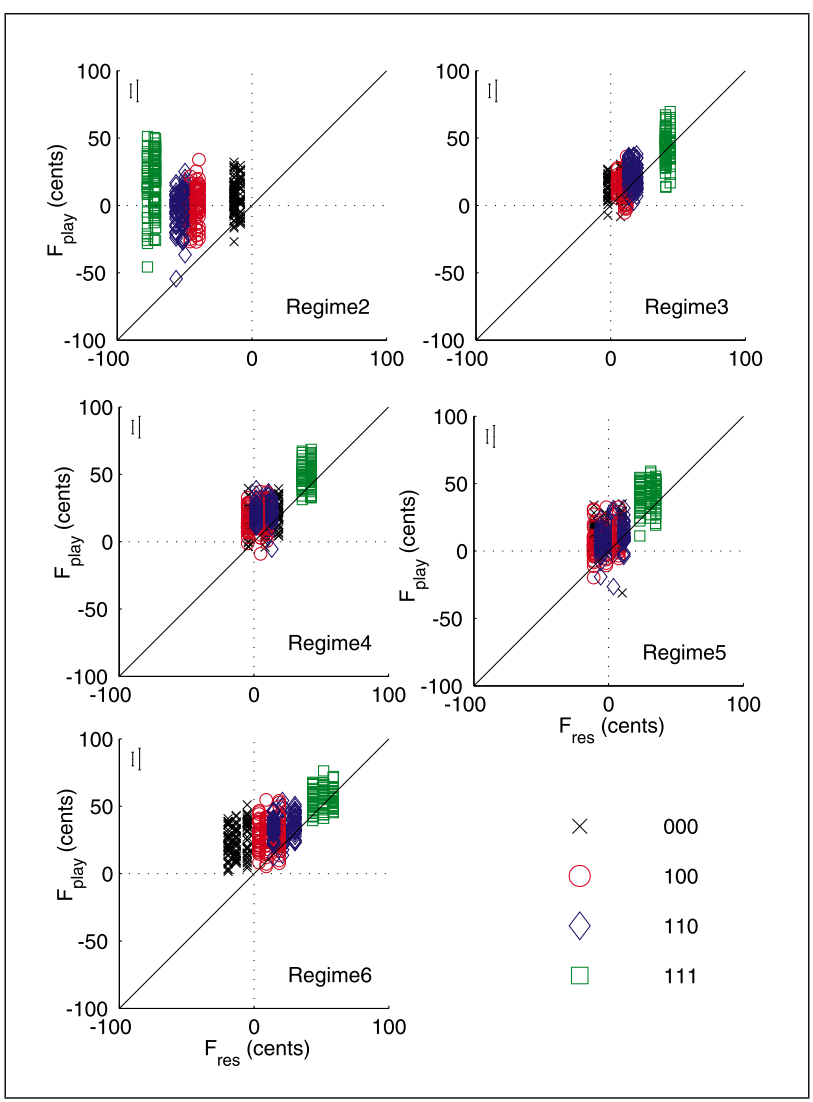

Figure 6. Playing frequencies $\left(\mathrm{F}_{\text {play }}\right)$ as functions of resonance frequencies $\left(\mathrm{F}_{\text {res }}\right)$, given in cents (taking the equally-tempered scale as a reference) for all the 2160 recorded notes. There is one figure for each regime, from regime 2 to regime 6 , and a different marker for each fingering: 000 in crosses, 100 in circles, 110 in diamonds and 111 in squares. The error bar on the left represents twice the average standard deviation of a note. The error bar on the right represents twice the average reproducibility of the trumpet players.

thus calculated on these 9 notes and then the mean of these standard deviations is calculated on all the notes played by all the musicians (it is in fact a mean on 240 standard deviations). This error is equal to 8 cents, which is more important than the average standard deviation. Indeed, this reproducibility is calculated by considering the three dynamic levels, which leads to more important variations of playing frequency. It corresponds to an audible pitch difference. Indeed, the just-noticeable difference (JND) is about $3 \mathrm{~Hz}$ for sine waves and $1 \mathrm{~Hz}$ for complex tones below $500 \mathrm{~Hz}$. Above $1000 \mathrm{~Hz}$, the JND for sine waves is about 10 cents $[38,39,40]$. Standard deviations $\sigma_{1}$ and $\sigma_{2}$ can also be calculated for each regime or for each trumpet player. The results are presented in Tables III and IV. Table III shows that regime 2 induces more variation on both the varying playing frequency of the note and the reproducibilty of the musicians. Table IV shows that players have more or less the same reproducibility even if player 4 seems to play more straight notes and is more repeatable than the others. 
Table III. Average standard deviation $\sigma_{1}$ and average reproducibility of players $\sigma_{2}$ calculated for each regime (in cents).

\begin{tabular}{|ccccccc|}
\hline Regime & 2 & 3 & 4 & 5 & 6 & All \\
\hline$\sigma_{1}$ & 8 & 5 & 4 & 4 & 3 & $\mathbf{5}$ \\
$\sigma_{2}$ & 11 & 7 & 7 & 8 & 8 & $\mathbf{8}$ \\
\hline
\end{tabular}

Table IV. Average standard deviation $\sigma_{1}$ and average reproducibility of players $\sigma_{2}$ calculated for each trumpet player (in cents).

\begin{tabular}{|cccccc|}
\hline Trumpet player & 1 & 2 & 3 & 4 & All \\
\hline$\sigma_{1}$ & 5 & 5 & 6 & 3 & $\mathbf{5}$ \\
$\sigma_{2}$ & 10 & 8 & 8 & 7 & $\mathbf{8}$ \\
\hline
\end{tabular}

In Figure 6, for each regime, there are 12 columns of points that represent all the combinations of the 4 fingerings for the 3 trumpets (a column is located at the value of the resonance frequency of the regime). For each column, there are 36 points that represent the notes played 3 times by the 4 musicians for the 3 dynamic levels.

The results show first that for all the regimes, the range of the data is important. Indeed, playing frequencies extend over 50 cents in average (and even more for the second regime). Secondly, for all the regimes, the playing frequency is higher than the resonance frequency (points are almost all above the line of equation $\mathrm{F}_{\text {play }}=\mathrm{F}_{\text {res }}$ ). In particular, the playing frequencies of the second regime are shifted up to the greatest extent with respect to the resonance frequencies. This observation can be related to the inharmonicity of the resonances corresponding to the second regime, which were observed to be too low in Figure 2. For the 111 fingering in particular, where the inharmonicity is high, we notice that there is a "compensation phenomenon" for the playing frequency, which is much higher than the resonance. This may be due to the coupling musician/instrument, or just to the musician. Notes played with this fingering are thus located in the three leftmost columns. On the other hand, for short tubes, as for the 000 fingering, the playing frequencies are quite close to the bore resonance frequencies. For regimes 3 to 5 , playing frequencies are, in average, close to the resonance frequencies. Finally, for the sixth regime, playing frequencies seem to be somewhat higher than resonance frequencies, especially for the 000 fingering. This figure is interesting to visualise the raw data, but we need to define a reference for each player to draw more precise conclusions.

\subsection{Models of the playing frequency}

The objective of this section is to estimate to what extend the resonance frequency can be used to predict the playing frequency. Different linear models can be proposed to predict the value of the playing frequency Fplay. The simplest model than can be proposed is

$$
\text { Fplay }_{i j k l m}=\text { Fres }_{k m}+\varepsilon_{k m},
$$

where Fplay $i_{i j k l m}$ is the value of the measured playing frequency for musician $(i=1 \ldots$ I with $\mathrm{I}=4$, see Section 4.2 for more details on the variables), dynamics $(j=1 \ldots \mathrm{J}$ with $\mathrm{J}=3)$, trumpet $(k=1 \ldots \mathrm{K}$ with $\mathrm{K}=3)$, fingering $(l=1 \ldots \mathrm{L}$ with $\mathrm{L}=4)$ and regime $(m=1 \ldots \mathrm{M}$ with $M=5)$, Fres $_{k m}$ is the value of the measured resonance frequency for trumpet $k$ and regime $m$ and $\varepsilon_{k m}$ is the error term.

In this case, the predicted value of the playing fre-

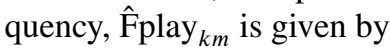

$$
\hat{\text { Fplay }}_{k m}=\text { Fres }_{k m} \text {. }
$$

To estimate the quality of the model, two classical indicators can be computed [41],

- The mean square error MSE of the model. It quantifies the difference between the observed value and the value predicted by the model:

$$
\mathrm{MSE}=\frac{1}{\mathrm{I} * \mathrm{~J} * \mathrm{~K}^{*} \mathrm{~L} * \mathrm{M}} \sum_{i, j, k, l, m}\left(\text { Fplay }_{i j k l m}-\hat{\mathrm{Fplay}}_{k m}\right)^{2} .
$$

- The MAPE (Mean Absolute Percentage Error). It is a measure of accuracy of a method for constructing fitted series values in statistics. It usually expresses accuracy as a percentage:

$$
\text { MAPE }=\frac{100 \%}{\mathrm{I}^{*} \mathrm{~J} * \mathrm{~K} * \mathrm{~L} * \mathrm{M}_{i, j, k, l, m}} \sum_{\mid}\left|\frac{\text { Fplay }_{i j k l m}-\hat{\text { Fplay }}_{k m}}{\text { Fplay }_{i j k l m}}\right| .
$$

Several models can be fitted to the data, from the simplest to the more complex, taken the different factors of the experiments into account. Four models are thus defined as follows:

$$
\begin{aligned}
& \text { Model 1: } \quad \hat{F}_{\text {play }}{ }_{k m}=\text { Fres }_{k m} \text {, } \\
& \text { Model 2: } \quad \hat{\text { Fplay }}_{k m}=a \text { Fres }_{k m} \text {, } \\
& \text { Model 3: } \quad \hat{F} \mathrm{play}_{k m}=a \text { Fres }_{k m}+\alpha_{i} \text {, } \\
& \text { Model 4: } \quad \text { Fplay }_{k m}=a \text { Fres }_{k m}+\alpha_{i}+\beta_{j} \text {, }
\end{aligned}
$$

where $a$ is the coefficient of the regression, $\alpha_{i}$ represents the effect of the musician and $\beta_{j}$ represents the effect of the dynamics. A simple linear regression is used to estimate the coefficient $a$ (Model 2), and analysis of covariance (ANCOVA) is used for Model 3 and 4 to estimate conjointly the coefficient $a$ and the parameters $\alpha_{i}$ and $\beta_{j}$.

Results in Table $\mathrm{V}$ indicate that, on average, the percentage of error of the four models is around 1\%. Even for the more complex model, Model 4, which takes all the experimental factors into account, the average error is around $1 \%$. These results indicate that it is not possible to predict the playing frequency from the resonance frequency with an average accuracy error lower than $1 \%$, which is 16 cents. This is more than the noticeable difference in pitch.

The introduction of the dynamic level and the musician in Model 4 does not give a significant improvement of the model quality: the MSE decreases, which is normal since it is a least square procedure, but the MAPE increases lightly from Model 2 to Model 4. 
Table V. Estimation of the quality of the four models with the MSE and MAPE.

\begin{tabular}{|lccc|}
\hline & MSE & MAPE & coeff a \\
\hline Model 1 & 101.03 & $1.18 \%$ & \\
Model 2 & 87.28 & $0.96 \%$ & 1.0085 \\
Model 3 & 86.69 & $0.979 \%$ & 1.0097 \\
Model 4 & 86.23 & $0.993 \%$ & 1.0111 \\
\hline
\end{tabular}

\section{Quantification of the discrepancy be- tween playing frequencies and resonance frequencies}

\subsection{Histogram of the distribution of the playing frequency}

In order to have a global view on all the 2160 played notes, it is possible to represent the data into a bar graph, as shown on Figure 7(a). In that histogram, each playing frequency is given in cents, taking its corresponding resonance frequency as a reference. The results seem to be normally distributed somewhat around +20 cents, but there are some abnormally high played notes around +100 cents. These notes in fact correspond to the second regime since, as we saw in section 5.1, for fingerings involving a long cylindrical part in the trumpet, playing frequencies are much higher than resonance frequencies. By removing all notes from regime 2, as shown in Figure 7(b), results seem to better fit a normal distribution. The sampling distribution of mean $\mu$ is equal to 15.5 cents and the standard deviation $\sigma$ is equal to 12.7 cents. A $95 \%$ confidence interval can be determined with a normal distribution, given the size of our samples (thousands of observations) and the central limit theorem [42]. The $95 \%$ confidence for the mean is thus $[\mu-1.96 \sigma / \sqrt{n} \quad \mu+1.96 \sigma / \sqrt{n}]$, with $n$ the number of samples. The mean $\mu$ therefore ranges from 14.3 to 16.7 cents. If we include the second regime in the data, $\mu$ then ranges from 19.9 to 23.3 cents.

This representation of the results shows us that playing frequencies are usually around 15 to 20 cents higher than the resonance frequencies, taking the temperature into account. We must notice that the fact that the value of $\mu$ is of the same order than the average accuracy error in estimating the playing frequency from the resonance frequency with models from Section 5.2 is a coincidence. These two quantities represent two different things: the MAPE (around 1\% - 16 cents), is the average prediction error of the playing frequency modelled with ANCOVA and linear regression, whereas $\mu$ is the average deviation of the playing frequency from the resonance frequency. For all the regimes, the error margin of the $95 \%$ confidence interval is 1.7 cents. By removing the second regime this error drops to 1.2 cents.

Nevertheless, these are absolute results whereas instrument makers are generally more interested in relative results. Indeed, a craftsman does not want his instrument to play defined frequencies, especially since players can tune their instruments in several ways. So, his interest is

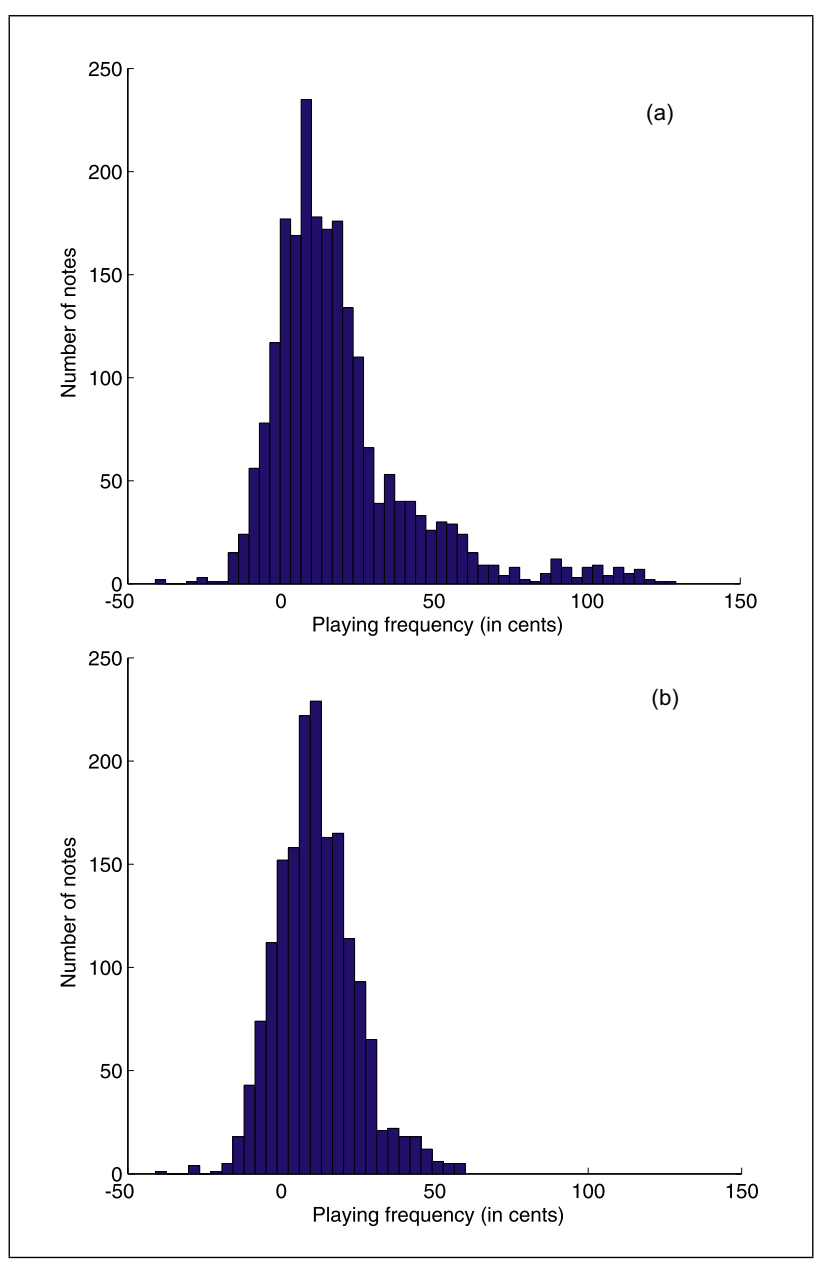

Figure 7. (a) Histogram representing all the 2160 playing frequencies and (b) Histogram representing the same data but without the second regime. Data are expressed in cents, as a difference between each playing frequency and its corresponding resonance frequency.

to make an instrument that can play intervals in tune. Consequently, it is useful to study differences of playing frequencies instead of the frequencies themselves.

\subsection{Differences of playing frequencies vs differences of resonance frequencies}

In order to study differences, references need to be chosen: one reference for the resonance frequencies (for each trumpet), and one reference for the playing frequencies (for each couple musician and trumpet). Concerning the resonance frequencies, we propose to consider the fourth regime of the fingering 000 (corresponding to the concert note $\mathrm{Bb} 4$ ) as the 0 cent reference. For the playing frequencies, we propose to consider the empirical mean of the frequency of the played note $\mathrm{Bb} 4$ as the 0 cent reference. This average is calculated on the 3 repetitions of the note $\mathrm{Bb} 4$ played mezzo forte by a musician on each trumpet. There are consequently 12 different references (3 trumpets and 4 musicians). This way of defining a reference is in fact logical, because the note chosen to serve as a reference 


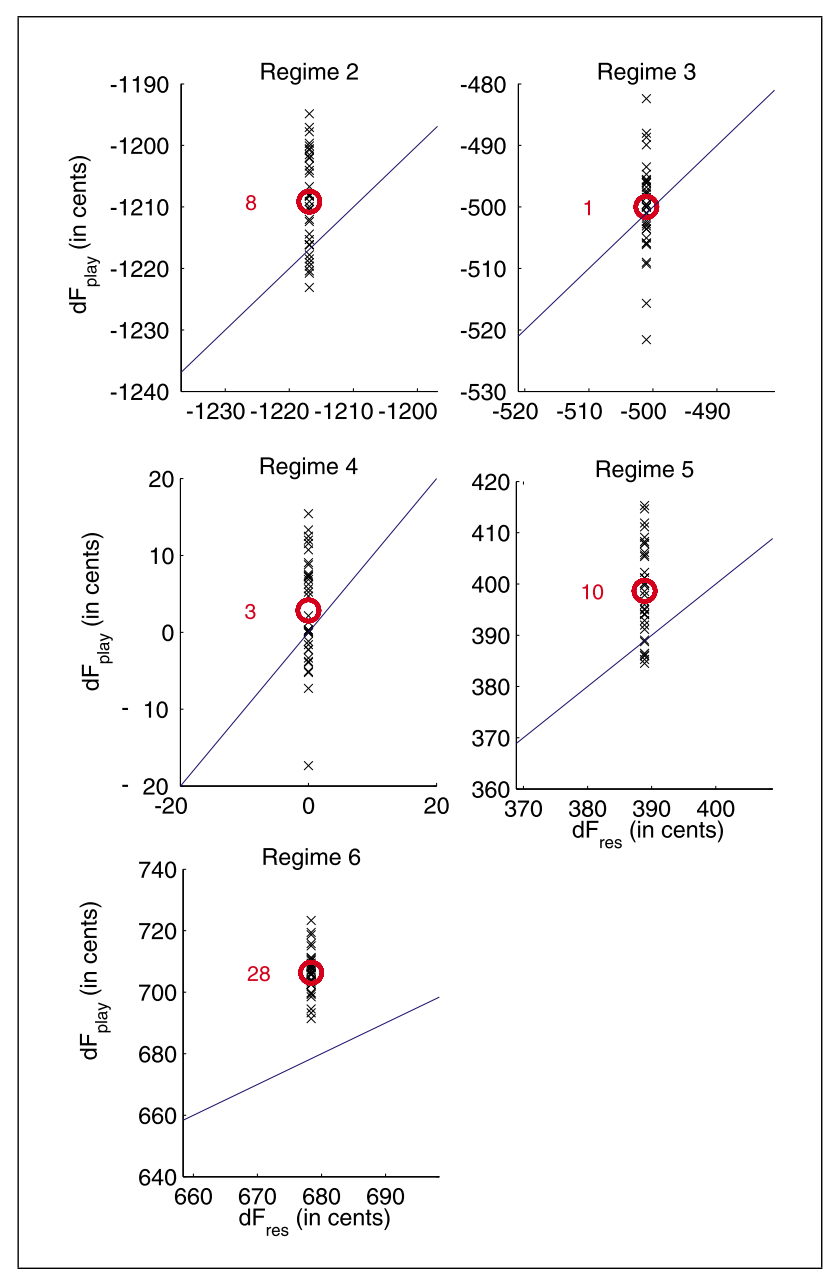

Figure $8 . \mathrm{dF}_{\text {play }}$ as function of $\mathrm{dF}_{\text {res }}$ (in crosses) for regimes 2 to 6 played by all the musicians on the trumpet CHMQ for the 000 fingering. Circles represent the average playing frequency and the written numbers give the distance, in cents, from this mean to the line representing $\mathrm{dF}_{\text {play }}=\mathrm{dF}_{\text {res }}$.

corresponds to the tuning note generally used by trumpet players to tune their instrument.

Figure 8 thus presents the differences of playing frequencies as function of the differences of resonance frequencies for the 000 fingering of CHMQ trumpet. These differences are given in cents, taking the references defined above into account. The average of all the playing frequencies is also given for each regime, represented with a circle and its distance, in cents, to the line of equation $\mathrm{dF}_{\text {play }}=\mathrm{dF}_{\text {res }}$ is indicated. First, it is important to notice that, even if the fourth regime of that fingering is taken as a reference, the average deviation is +3 cents and not zero for that note. This is due to a discrepancy between the frequencies of a same note played with the different dynamic levels (piano and forte). Then the deviation of other regimes is +8 cents (regime 2 ), +1 cent (regime 3 ), +10 cents (regime 5 ) and +28 cents (regime 6 ). For the regimes 2 to 5 , the deviation is very weak, in the same range as the uncertainty in the determination of the playing frequency and the repeatability of the musicians (see Section 5.1). For these 4 regimes, it is thus possible to conclude that in

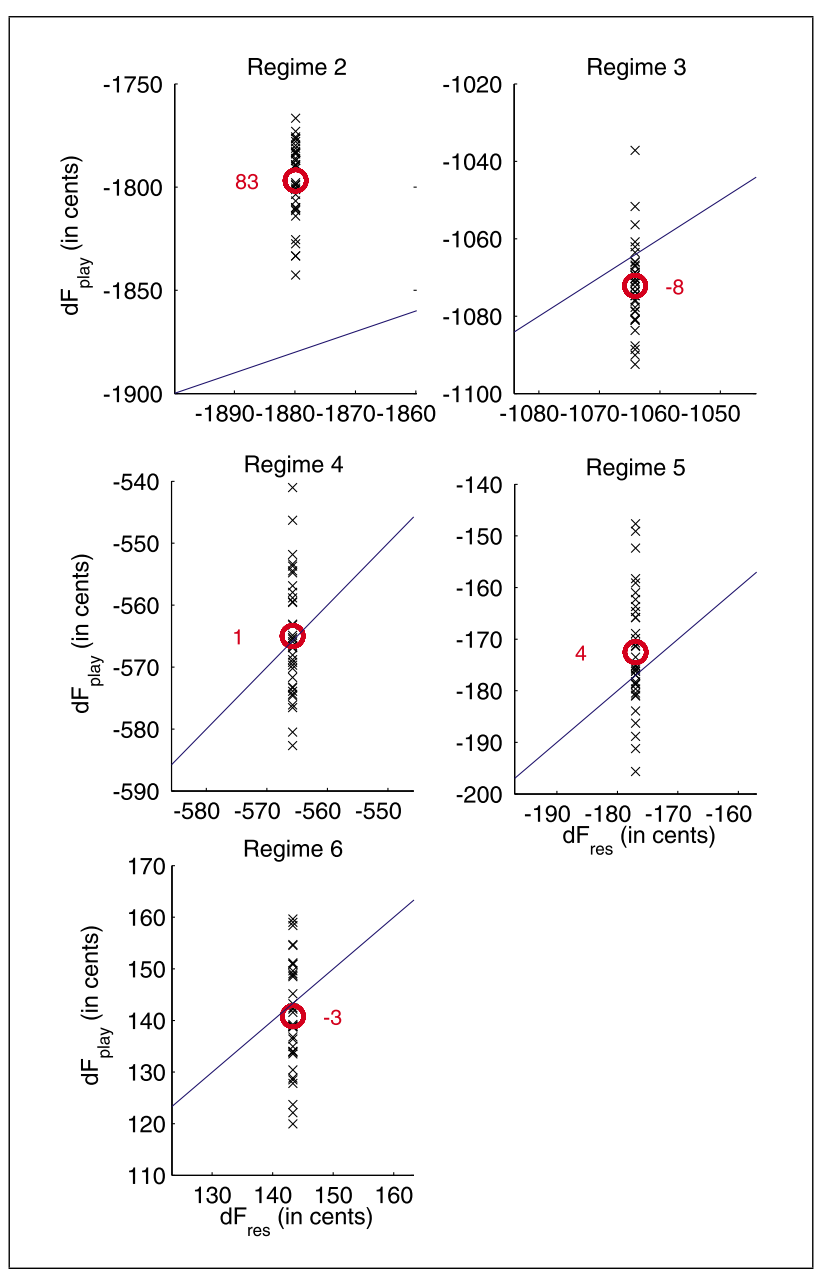

Figure 9. $\mathrm{dF}_{\text {play }}$ as function of $\mathrm{dF}_{\text {res }}$ (in crosses) for regimes 2 to 6 played by all the musicians on the trumpet CHMQ for the 111 fingering. Circles represent the average playing frequency and the written numbers give the distance, in cents, from this mean to the line representing $\mathrm{dF}_{\text {play }}=\mathrm{dF}_{\text {res }}$.

average, a variation of the resonance frequency leads to a variation of the playing frequency in the same order of magnitude. For the sixth regime, a variation of the resonance frequency leads to a much higher variation of playing frequency, which is a surprising unexpected result.

It has to be noticed that these conclusions represent only an average behaviour of the instrument: by observing the total variability of the playing frequency, we remark that the data spreads out over about 30 cents for each regime. This variability is inherent to the trumpet playing, where several uncontrolled factors may modify the playing frequency of notes.

Figure 9 then shows the same kind of plot but for the 111 fingering of CHMQ trumpet. This time, regimes 3 to 6 are well centred on the line of equation $\mathrm{dF}_{\text {play }}=\mathrm{dF}_{\text {res }}$, whereas regime 2 gives variations of playing frequency much higher than variations of resonance frequency. Indeed, it was explained in section 5.1 that the longer the cylindrical pipe, the more inharmonic the second resonance is. Moreover, for the second regime data are even more spread out than for other regimes (about 70 cents). 
Table VI. Deviation of the average of all the $\mathrm{dF}_{\text {play }}$ for each regime of each fingering on each trumpet to the line of equation $\mathrm{dF}_{\text {play }}=\mathrm{dF}_{\text {res }}$ (in black) and the line of equation $\mathrm{dF}_{\text {play }}=\mathrm{dSF}$ (in grey, these are results from section 6.3), given in cents with the fourth regime of 000 fingering taken as a reference.

\begin{tabular}{|c|c|c|c|c|c|c|c|c|c|c|c|}
\hline \multirow{4}{*}{$\begin{array}{c}\text { Fingering } \\
000\end{array}$} & \multirow{4}{*}{$\begin{array}{l}\text { Trumpet } \\
\text { CHMQ } \\
\text { DKNR } \\
\text { NORM }\end{array}$} & \multicolumn{2}{|c|}{2} & \multicolumn{4}{|c|}{$\begin{array}{c}\text { Regime } \\
4\end{array}$} & \multicolumn{2}{|c|}{5} & \multicolumn{2}{|c|}{6} \\
\hline & & 8 & 5 & 1 & 13 & 3 & 3 & 10 & 12 & 28 & 34 \\
\hline & & 15 & 10 & 4 & 11 & 3 & 3 & 6 & 7 & 33 & 39 \\
\hline & & 6 & 9 & -2 & 7 & 4 & 4 & 7 & 10 & 24 & 29 \\
\hline & CHMQ & 35 & -7 & -3 & 1 & 6 & 10 & 2 & 2 & 11 & 13 \\
\hline 100 & DKNR & 41 & -16 & 2 & -5 & 9 & 10 & 2 & 2 & 11 & 13 \\
\hline & NORM & 24 & 13 & -7 & -4 & 3 & 8 & -1 & $\mathbf{0}$ & 7 & 9 \\
\hline & CHMQ & 44 & 19 & -6 & -7 & 2 & 8 & -1 & $\mathbf{0}$ & 3 & 5 \\
\hline 110 & DKNR & 51 & -27 & 4 & -2 & 8 & 17 & 1 & 1 & 6 & 8 \\
\hline & NORM & 32 & -21 & -7 & -6 & 3 & 8 & -5 & -3 & 1 & 3 \\
\hline & CHMQ & 83 & -35 & -8 & -10 & 1 & 2 & 4 & 6 & -3 & -3 \\
\hline 111 & DKNR & 90 & -38 & 1 & -4 & 6 & 4 & 6 & 11 & -3 & -4 \\
\hline & NORM & 72 & -39 & -14 & -12 & -4 & -5 & -1 & 4 & -6 & -6 \\
\hline
\end{tabular}

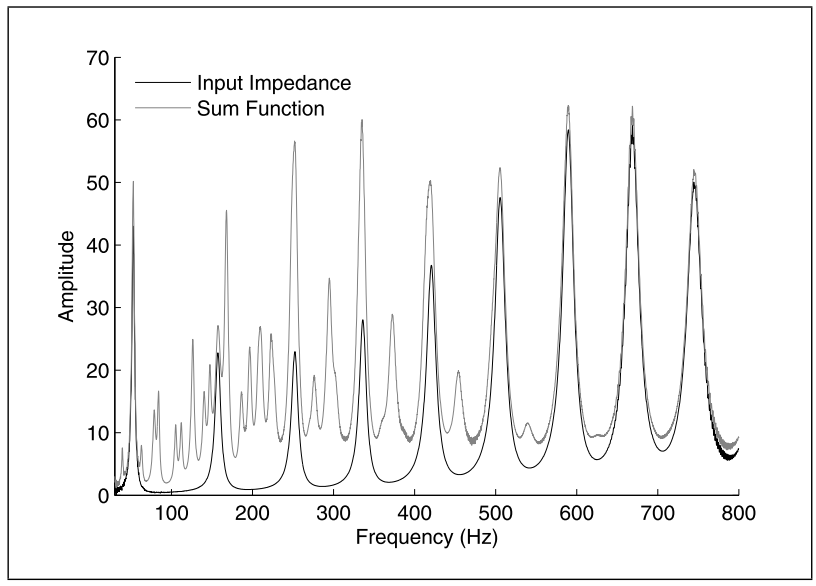

Figure 10. Comparison between the measurement of the input impedance amplitude of the CHMQ trumpet with 111 fingering (in black) with the sum function calculated from this impedance with equation 10 (in grey).

The results for all the fingerings on all the trumpet are given in Table VI. The order of magnitude of the distances are the same whatever the trumpet. This table shows that it is possible to consider that a variation of resonance frequency leads in average to a variation of the playing frequency of the same order for regimes 3 to 6 of all fingerings, except regime 6 of 000 fingering. While regimes 3 to 5 have almost constant variations of playing frequency over the different fingerings, regimes 2 and 6 have a completely different behaviour. For regime 6 , a variation of resonance frequency first leads to a higher variation of playing frequency for fingering 000. Then, the deviation between $\mathrm{dF}_{\text {play }}$ and $\mathrm{dF}_{\text {res }}$ decreases when the first two valves are depressed. Finally, for the 111 fingering, the variation of playing frequency becomes smaller than the variation of resonance frequency. For the second regime it is the contrary, $\mathrm{dF}_{\text {play }}$ differs more and more from $\mathrm{dF}_{\text {res }}$ as the cylindrical part of the trumpet gets longer. This result was expected as it has already been observed in section 5.1. The fact that playing frequencies for regime 6 are much higher only for the 000 fingering is not an expected result.

We have seen that the variations of resonance frequencies are a good indicator of the playing frequencies variations but we also experienced some discrepancies for the second and the sixth regime. In literature [19, 18], other indicators like "sum functions", have been defined in order to predict playing frequencies more accurately than the resonance frequencies from the input impedance. A sum function is thus evaluated by using our set of experimental data in the following section.

\subsection{The "sum function": a supposed indicator of the playing frequency}

Wogram [19] (who was quoted later by Pratt and Bowsher [18]) introduced what he termed a "Summenprizinzip" (or "sum function" in English): the impedance values of an instrument at integral multiples of the fundamental frequency combine at the player's lips to establish the playing frequency. Actually, the sum function is the sum of the acoustic power entering the resonator for a forced oscillation with fixed flow rate amplitude and spectrum. In lip reed instruments, the playing frequency strongly depends also on the reed natural frequency which is not taken into account in that function. One version of the sum function can be calculated as

$$
S(f)=\sum_{i=1}^{n} \frac{1}{i} \operatorname{Re}[Z(i f)] .
$$

in which $n$ is maximized such that $n f<f_{\max }$, the highest frequency for which $Z$ is known.

An example of this sum function is given in Figure 10. This function is thus supposed, as claimed by Wogram, to predict the playing frequencies with a better accuracy than the resonance frequencies from the input impedance. 


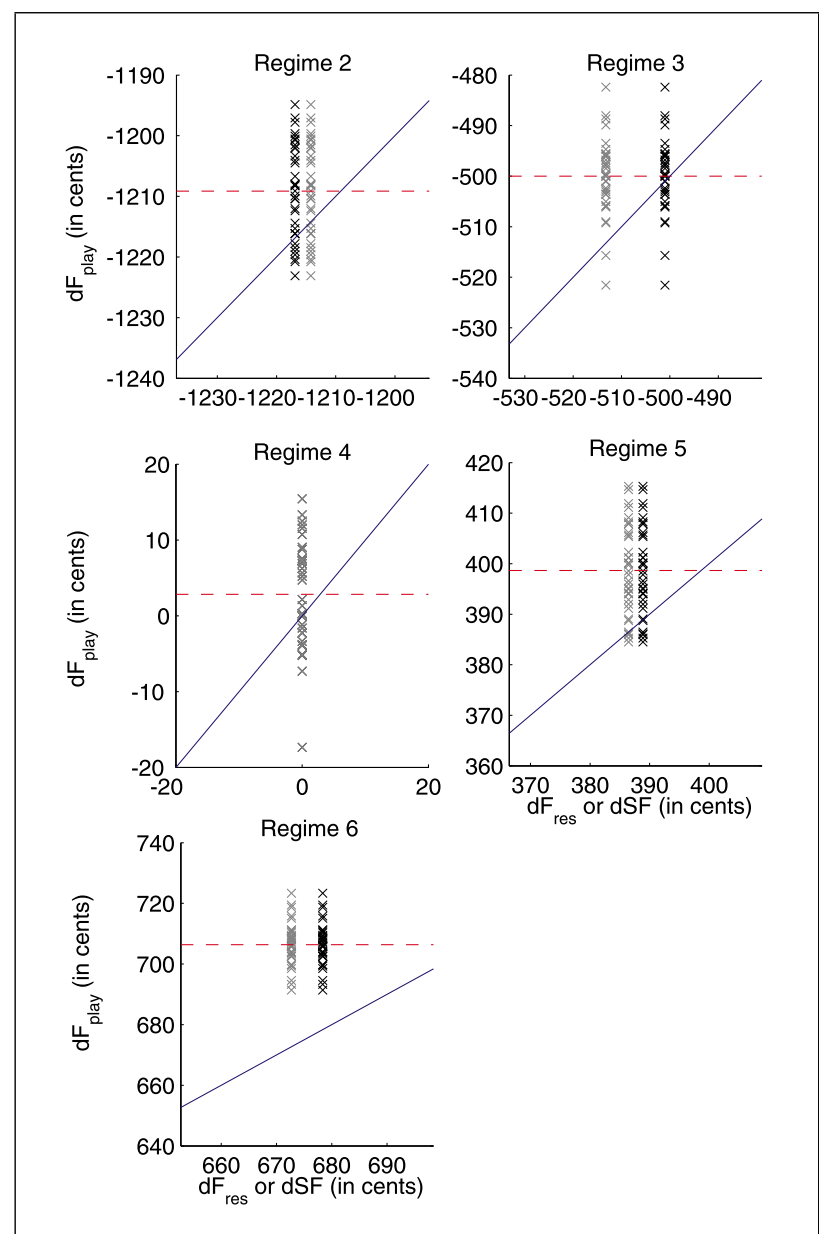

Figure 11. $\mathrm{dF}_{\text {play }}$ as function of $\mathrm{dF}_{\text {res }}$ (in black crosses) or $\mathrm{dSF}$ (in grey crosses) for regimes 2 to 6 played by all the musicians on the trumpet CHMQ for the 000 fingering. Dotted line represents the average playing frequency and straight line is the line of equation $\mathrm{dF}_{\text {play }}=\mathrm{dF}_{\text {res }}$ or $\mathrm{dF}_{\text {play }}=\mathrm{dSF}$.

In order to study if the sum function is able to predict the playing frequencies more accurately that the input impedance, we plot again $\mathrm{dF}_{\text {play }}$ as function of $\mathrm{dF}_{\text {res }}$ and as function of variations of the sum function peaks in Figures 11 and 12. As previously done for the resonance frequencies of the input impedance, a reference is taken: the frequency of the sum function peak corresponding to the regime 4 . Then, each peak of the sum function is given in cents, by calculating the difference with that reference, and is written dSF.

Figures 11 and 12 show how variations of the resonance frequencies taken from both the input impedance and the sum function are able to predict the variations of the playing frequency. Deviations of the average $\mathrm{dF}_{\text {play }}$ from $\mathrm{dF}_{\text {res }}$ and dSF are summarised in Table VI. As pointed out in the previous sections, there is a large discrepancy between $\mathrm{dF}_{\text {play }}$ and $\mathrm{dF}_{\text {res }}$ for the second regime of the 111 fingering. That is a reason why the sum function has been implemented and Figures 11 and 12 as well as Table VI show that, for this regime, $\mathrm{dSF}$ is closer to $\mathrm{dF}_{\text {play }}$ than $\mathrm{dF}_{\text {res }}$. Nevertheless, the sum function actually shifts the resonance

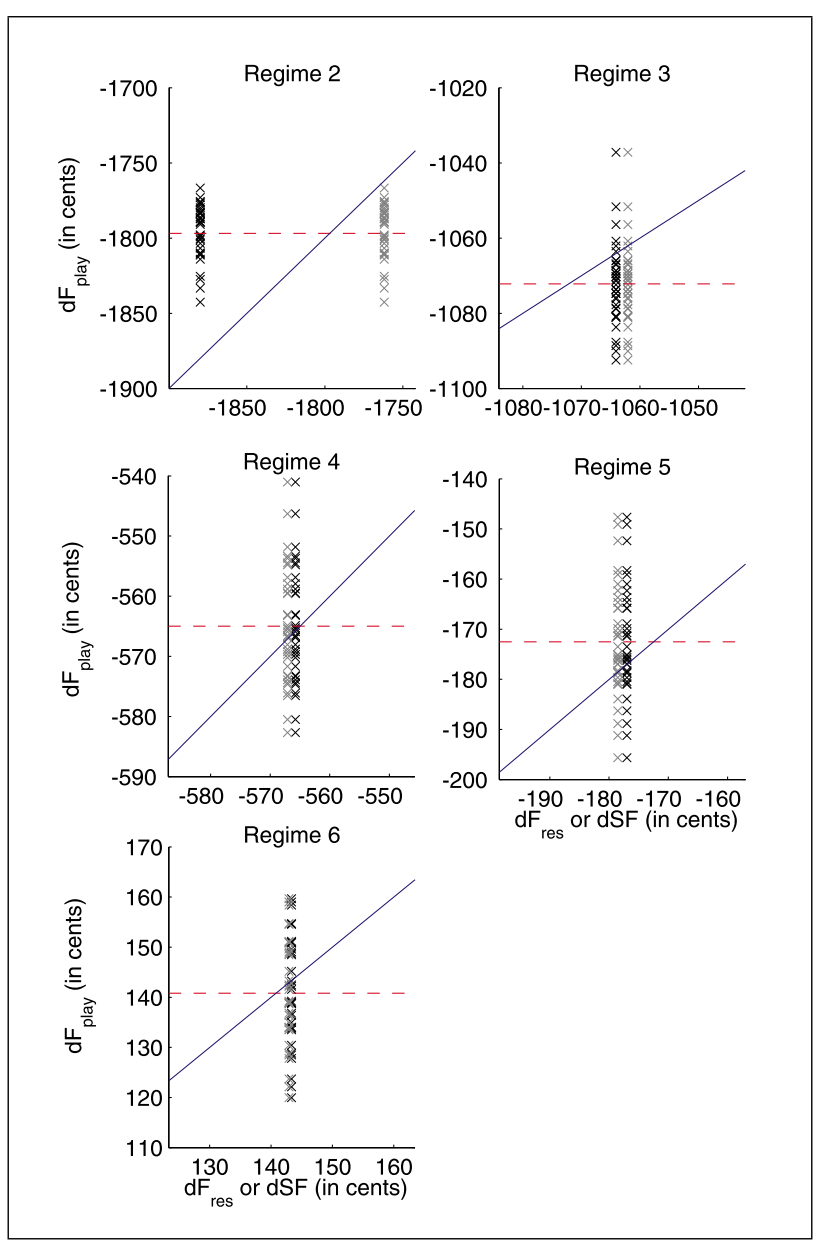

Figure 12. $\mathrm{dF}_{\text {play }}$ as function of $\mathrm{dF}_{\text {res }}$ (in black crosses) or $\mathrm{dSF}$ (in grey crosses) for regimes 2 to 6 played by all the musicians on the trumpet CHMQ for the 111 fingering.Dotted line represents the average playing frequency and straight line is the line of equation $\mathrm{dF}_{\text {play }}=\mathrm{dF}_{\text {res }}$ or $\mathrm{dF}_{\text {play }}=\mathrm{dSF}$.

frequencies to the right direction but it over-corrects the discrepancy. Moreover, for the other regimes, the input impedance allows predicting variations of resonance frequencies closer to those of playing frequencies than the peak frequencies of the sum function.

Consequently, the sum function does not seem to give more information on the playing frequencies than the simple input impedance.

\section{Conclusion}

This study proposed a quantitative assessment of the relations between the bore resonance frequencies and the playing frequencies, based on experiments made on three trumpets with four musicians for a large number of notes (different regimes, fingerings and dynamic levels). Even if it was already known that playing frequencies are close to bore resonance frequencies, no detailed work had previously been carried out to quantify it.

First, this study shows that the dynamic level does not have a strong influence on the playing frequencies and that 
the four musicians have relatively the same "global" behaviour, as they all play on average in the order of 8 to 20 cents above the bore resonance frequencies.

Second, a closer analysis of the data shows that the average standard deviation of the playing frequency is about 5 cents, which means that a played note is stable with an uncertainty of 5 cents. Furthermore, the average repeatability of a musician, calculated on his 9 repetitions of a same note, is about 8 cents. Therefore, there is no need to find a predictor of the playing frequency more accurate than 8 cents.

Then, by representing the played notes as an histogram it is possible to conclude that, from regime 3 to 6 , playing frequencies are in average 15 cents higher than the resonance frequencies. The error margin on the estimation of that mean is 1.2 cents at a $95 \%$ confidence level.

Finally, by examining differences instead of just frequencies themselves, the impact of the musicians' behaviour is diminished. Moreover, craftsmen often work by making small changes in the geometry of their instruments and studying the differences induced by the modification. So, focusing on differences is a way to get closer to the craftsman's process.

Regime 4 played with the 000 fingering is thus taken as a reference to calculate those differences, since it is the note generally used to tune the instruments. Results show that a variation of bore resonance frequency leads in average to a variation of playing frequency of the same order for regime 3 to 6 (but surprisingly, except the sixth regime for the 000 fingering). For regime 2, this rule is not satisfied because the notes are played at a higher frequency than the bore resonance. The inharmonicity of the notes of regime 2 could be a reason to explain this behaviour. These results might show that the inharmonicity plays a role on the control of the playing frequencies. It should indeed be possible that, when the bore resonance frequency corresponding to the played note is in an harmonic relationship with the other resonances, a variation of the resonance frequency leads to a variation of the playing frequency of the same range. On the other hand, when the bore resonance frequencies are inharmonic, that relation is not valid any more. This is shown in the study of Dalmont et al. [43] for one saxophone fingering. Nevertheless, further analysis is required to support this explanation.

An attempt was made to model this effect with the sum function. For the second regime, played frequencies are actually closer to the sum function peaks frequencies than to the bore resonances. Nevertheless, a discrepancy still exists and the prediction of the sum function is less accurate for other regimes. In conclusion, the sum function does not seem to be more relevant than the input impedance in order to predict playing frequencies. The resonance frequency is thus a good objective indicator for predicting the playing frequency, as it does not take the influence of the musician into account. This is interesting for craftsmen whose instruments need to be played by virtual musicians, and who often proceed by small adjustments on their instruments.
Our results are obtained with three particular trumpets that do not represent all the possible trumpets in the market. We must refrain any generalization of the results to the trumpet in general, further studies are needed to prove the robustness of the relationship playing frequency/resonance frequency.

Also, for further work, it will then be interesting to compare these results with measurements using an artificial mouth [44] and simulations.

\section{Acknowledgement}

This research was funded by the French National Research Agency ANR within the PAFI project (Plateforme d'Aide à la Facture Instrumentale in French). The authors would like to thank all the trumpet players who participated in this study as well as A. Burke, K. Cedergren, J.-P. Dalmont, D. Lopatin, A. Mamou-Mani and R. Piéchaud for proofreading and valuable talks.

\section{References}

[1] J. Backus: Input impedance curves for the brass instruments. J. Acoust. Soc. Am. 60 (1976) 470-480.

[2] R. Caussé, J. Kergomard, X. Lurton: Input impedance of brass musical instruments - Comparison between experiment and numerical models. J. Acoust. Soc. Am. 75 (1984) 241-245.

[3] P. Eveno, J.-P. Dalmont, R. Caussé, J. Gilbert: Wave propagation and radiation in a horn: comparisons between models and measurements. Acta Acustica united with Acustica 98 (2012) 158-165.

[4] C. A. Macaluso, J.-P. Dalmont: Trumpet with near-perfect harmonicity: design and acoustic results. J. Acoust. Soc. Am. 129 (2011) 404-414.

[5] J.-P. Dalmont: Acoustic impedance measurement. Journal of Sound and Vibration 243 (2001) 427-459.

[6] P. Dickens, J. Smith, J. Wolfe: Improved precision in measurements of acoustic impedance spectra using resonancefree calibration loads and controlled error distribution. J. Acoust. Soc. Am 121 (2007) 1471-1481.

[7] J. Gilbert, J. Kergomard, E. Ngoya: Calculation of the steady-state oscillations of a clarinet using the harmonic balance technique. J. Acoust. Soc. Am. 86 (1989) 35-41.

[8] S. Farner, C. Vergez, J. Kergomard, A. Lizée: Contribution to harmonic balance calculations of self-sustained periodic oscillations with focus on single-reed instruments. J. Acoust. Soc. Am. 119 (2006) 1794-1804.

[9] E. Poirson, J.-F. Petiot, J. Gilbert: Study of the brightness of trumpet tones. J. Acoust. Soc. Am. 118 (2005) 2656-2666.

[10] J. S. Cullen, J. Gilbert, D. Campbell: Brass instruments: Linear stability analysis and experiments with an artificial mouth. Acta Acustica united with Acustica 86 (2000) 704 724.

[11] S. Bilbao: Numerical sound synthesis. Finite difference schemes and simulation in musical acoustics. Wiley, 2009, Ch. Acoustic tubes, 249-286.

[12] L. Trauntmann, R. Rabenstein: Digital sound synthesis by physical modeling using the functional transformation 
method. Kluwer Academic / Plenum publishers, 2003, Ch. Classical synthesis methods based on physical models, 6394.

[13] P. Guillemain, C. Vergez, D. Ferrand, A. Farcy: An instrumented saxophone mouthpiece and its use to understand how an experienced musician plays. Acta Acustica united with Acustica 96 (2010) 622-634.

[14] G. Scavone, A. Lefebvre, A. da Silva: Measurement of vocal-tract influence during saxophone performance. J. Acoust. Soc. Am. 123 (2008) 2391-2400.

[15] J.-M. Chen, J. Smith, J. Wolfe: Saxophonists tune vocal tract resonances in advanced performance techniques. J. Acoust. Soc. Am. 129 (2011) 415-426.

[16] T. Kaburagi, N. Yamada, T. Fukui, E. Minamiya: A methodological and preliminary study on the acoustic effect of a trumpet player's vocal tract. J. Acoust. Soc. Am. 130 (2011) 536-545.

[17] J.-M. Chen, J. Smith, J. Wolfe: Do trumpet players tune resonances of the vocal tract? J. Acoust. Soc. Am. 131 (2012) 722-727.

[18] R. Pratt, J. Bowsher: The objective assessment of trombone quality. Journal of Sound and Vibration 65 (1979) 521547.

[19] K. Wogram: Ein beitrag zur ermittlung der stimmung von blechblasininstrumenten (in english: A contribution to the measurement of the intonation of brass instruments). Dissertation. Technische Universität Carolo Wilhelmina, Braunschweig, 1972.

[20] M. Campbell, C. Greated: The musician's guide to acoustics. Schirmer Books, 1988, Ch. 9. Brass instruments, 303407.

[21] N. H. Fletcher, T. D. Rossing: The physics of musical instruments. Springer-Verlag, 1991, Ch. 14. Lip-driven Brass instruments, 365-393.

[22] A. Benade, D. Gans: Sound production in wind instruments. Ann. N.Y. Acad. Sci. 155 (1968) 247-263.

[23] E. Poirson, J.-F. Petiot, J. Gilbert: Integration of user perceptions in the design process: Application to musical instrument optimization. J. Mech. Des 129 (2007) 12061214.

[24] J.-F. Petiot, E. Poirson, J. Gilbert: Study of the relations between trumpets' sounds characteristics and the input impedance. Proceedings of Forum Acusticum 2005, Budapest, Hungary, 747-752.

[25] A. de Cheveigné, H. Kawahara: YIN, a fundamental frequency estimator for speech and music. J. Acoust. Soc. Am 111 (2002) 1917-1930.

[26] J. Gilbert, L. M. L. Ruiz, S. Gougeon: Influence de la température sur la justesse d'un instrument à vent (in english: Influence of the temperature on the intonation of a wind instrument). Proceedings of Congrès Français d'Acoustique 2006, Tours.

[27] D. Noreland: An experimental study of temperature variations inside a clarinet. Proceedings of the Stockholm $\mathrm{Mu}$ sic Acoustics Conference 2013, SMAC 2013, Stockholm, Sweden.
[28] J.-C. Le Roux: Le haut-parleur électrodynamique: estimation des paramètres électroacoustiques aux basses fréquences et modélisation de la suspension (in english: The electrodynamic loudspeaker: Estimate of the electroacoustic parameters at low frequencies and suspension modelling). Dissertation. Université du Maine, 1994.

[29] P. Eveno: L'impédance d'entrée pour l'aide à la facture des instruments de musique à vent : mesures, modèles et lien avec les fréquences de jeu (in english: The input impedance for the support of the musical instruments making: measurements, models and link with the playing frequencies). Dissertation. Université Pierre et Marie Curie, 2012.

[30] V. Fréour, G. Scavone: Acoustical interaction between vibrating lips, dowstream air column, and upstream airways in trombone performance. J. Acoust. Soc. Am. 134 (2013) 3887-3898.

[31] N. Fletcher: Excitation mechanisms in woodwind and brass instruments. Acustica 43 (1979) 63-72.

[32] C. Vergez, X. Rodet: Dynamical systems and physical models of trumpet-like instruments. Acta Acustica united with Acustica 86 (2000) 147-162.

[33] M. Campbell: Brass instruments as we know them today. Acta Acustica united with Acustica 90 (2004) 600-610.

[34] S. Adachi, M. Sato: Time-domain simulation of sound production in the brass instrument. J. Acoust. Soc. Am. 97 (1995) 3850-3861.

[35] S. Yoshikawa: Acoustical behaviour of brass player's lips. J. Acoust. Soc. Am. 97 (1995) 1929-1939.

[36] R. Christensen: Plane answers to complex questions: The theory of linear model. Springer Texts in Statistics, Springer, 2002, Ch. 7. Multifactor Analysis of Variance, 156-195.

[37] H. Scheffé: The analysis of variance. New York: Wiley, 1959.

[38] B. Kollmeier, T. Brand, B. Meyer: Springer handbook of speech processing. Springer, 2008, Ch. Perception of Speech and Sound, 65.

[39] T. Letowski: A note on the difference limen for frequency differentiation. J. Sound Vib. 85 (1982) 579-583.

[40] W. M. Hartmann: Pitch, periodicity, and auditory organization. J. Acoust. Soc. Am. 100 (1996) 3491-3502.

[41] J. Armstrong: Principles of forecasting: a handbook for researchers and practitioners. Kluwer Academic Publishers, Norwell, MA, 2001, Ch. 14. Evaluating forecasting methods, 443-472.

[42] R. Christensen: Plane answers to complex questions: The theory of linear model. Springer Texts in Statistics, Springer, 2002, Ch. E. Inference for One Parameter, 451458.

[43] J.-P. Dalmont, B. Gazengel, J. Gilbert, J. Kergomard: Some aspects of tuning and clean intonation in reed instruments. Applied Acoustics 46 (1995) 19-60.

[44] J. Gilbert, S. Ponthus, J.-F. Petiot: Artificial buzzing lips and brass instruments: Experimental results. J. Acoust. Soc. Am. 104 (1998) 1627-1631. 